\title{
Tissue turnover of collagen type I, III and elastin is elevated in the PCLS model of IPF and can be restored back to vehicle levels using a phosphodiesterase inhibitor
}

Niels Ulrik Brandt Hansen ${ }^{1,2^{*}}$, Morten Asser Karsdal ${ }^{1,2}$, Sarah Brockbank ${ }^{3}$, Simon Cruwys ${ }^{3}$, Sarah Rønnow ${ }^{1}$ and Diana Julie Leeming ${ }^{1}$

\begin{abstract}
Background: The aim of this study was to develop and validate a model for pulmonary fibrosis, using ex vivo tissue cultures of lungs from bleomycin treated animals, enabling the investigation of fibrosis remodeling using novel biomarkers for the detection of ECM protein fragments. The combination of in vivo and ex vivo models together with ECM remodeling markers may provide a translational tool for screening of potential treatments for IPF.

Methods: Twenty female Sprague-Dawley rats, twelve weeks of age, were administrated either two doses of bleomycin (BLM) $(n=14)$ or saline $(n=6)$ I.T., two days apart. Ten rats were euthanized at day seven and the remaining ten rats at day fourteen, after the last dose. Precision-cut lung slices (PCLS) were made and cultured for $48 \mathrm{~h}$.

Ten female Sprague-Dawley rats, twelve weeks of age, were administrated either two doses of BLM $(n=7)$ or saline $(n=3)$ I.T., two days apart. The rats were euthanized fourteen days after the last dose. PCLS were made and

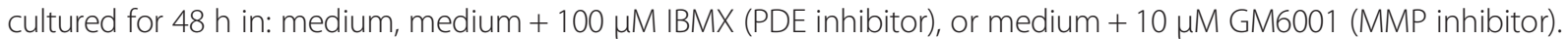

Turnover of type I collagen (P1NP, C1M), type III collagen (iP3NP, C3M) and elastin degradation (ELM7) was measured in the supernatant of the cultured PCLS.

Results: P1NP, C1M, iP3NP, C3M and ELM7 were significantly increased in supernatants from BLM animals ( $P \leq 0.05$ $P \leq 0.0001)$ when compared to controls. P1NP, C1M, IP3NP, C3M and ELM7 were significantly increased in supernatants from day seven BLM animals compared to day fourteen BLM animals ( $P \leq 0.05-P \leq 0.0001)$. P1NP, C1M, IP3NP, C3M and ELM7 were significantly decreased when adding IBMX to the culture medium of fibrotic lung tissue $(P \leq 0.05-P \leq$ $0.0001)$. C1M, C3M and ELM7 were significantly decreased when adding GM6001 to the culture medium ( $P \leq 0.05-$ $P \leq 0.0001)$.

Sirius Red and Orcein staining confirmed the presence of collagen and elastin deposition in the lungs of the animals receiving $B L M$.

Conclusions: The protein fingerprint technology allows the assessment of ECM remodeling markers in the BLM PCLS model. By combining in vivo, ex vivo models and the protein fingerprint technology in the fibrotic phase of the model, we believe the chance of translation from animal model to human is markedly increased.
\end{abstract}

Keywords: Precision-cut lung slices, ex vivo, IPF, Fibrosis, Biomarkers

\footnotetext{
* Correspondence: nuh@nordicbioscience.com

${ }^{1}$ Nordic Bioscience A/S, Herlev Hovedgade 205-207, 2730 Herlev, Denmark

University of Southern Denmark, SDU, Odense, Denmark

Full list of author information is available at the end of the article
} 


\section{Background}

Idiopathic interstitial pneumonias are a diverse group of diseases characterized by fibrosis and inflammation, of which idiopathic pulmonary fibrosis (IPF) is the most common type. The etiology of the disease is unknown but it is believed to be an interplay between genetic predispositions and environmental causes, such as pollution and cigarette smoke [1]. The hallmark of fibroproliferative diseases can be described as a dysregulated wound healing, leading to a shift in the balance of extracellular matrix (ECM) remodeling and accumulation of matrix components and end stage fibrosis with loss of function of the affected organ. The total ECM turnover is increased in IPF, meaning that both formation and degradation of proteins is upregulated, but the net result is matrix deposition. The formation and degradation of matrix proteins generate protein fragments, which are released into circulation, forming neoepitopes that may be measured as biomarkers. A paper just published in The Lancet describes how novel biomarkers of the ECM were able to separate fast progressing IPF patients from stable IPF patients, while some of the markers were also strongly predictive of overall survival [2]. The ECM has gained a lot of attention during the last decades and it is now recognized as a structure with multiple functions such as cell support, cytokine activation and signaling purposes [3]. It is known to be important for the phenotype and survival of cells, e.g. orientation of collagen can critically regulate cell and tissue behavior [4-6]. Moreover the ECM can serve as a storage site for multiple soluble/secreted cytokines and growth factors, thereby functioning as a repository. These factors can be released into the microenvironment upon matrix degradation by matrix metalloproteinases (MMPs) [7, 8]. The accumulation of ECM proteins also affects the rigidity of the matrix causing the matrix to become stiff. This stiffness affects the communication and signaling of the matrix and hence results in the thickening of the interstitium in the alveoli, which leads to less $\mathrm{O}_{2}$ uptake and shortness of breath. The stiffness has also been shown to affect myofibroblast activation which ultimately leads to more matrix deposition and rigidity, thus creating a positive feedback loop and linking matrix architecture and composition with cell phenotype [9-11].

The precision cut lung slice model using IPF tissue enables the possibility of studying the fibrotic processes in a multicellular system in which cell-cell, cell-ECM interactions and opened alveoli are preserved, thus maintaining the $3 \mathrm{D}$ structure seen in vivo $[12,13]$. The importance of the interplay between the cells and the microenvironment is very well described within the cancer field. Important factors such as the presence of all the different cell types, structural support from the ECM, signaling (e.g. via transmembrane receptors) and cytoskeletal and chromatin organization are some of the highlighted features. In order to get full insight into the biology of normal and diseased tissue, it is vital to create in vivo-like models for therapeutic testing to get the full context of the effects [14-17]. In addition, the PCLS model enables the study of a heterogeneous matrix, which provides an in vivo-like environment with minimum alterations of the natural conditions in order to study cell behavior and function. This is a very crucial matter when testing potential therapies [3]. Due to the importance and influence of the matrix in IPF, we used the fit for purpose Precision-Cut Lung Slice (PCLS) model [18] using in vivo BLM treated lung tissue combining it with a novel Protein Fingerprint biomarker technology for the assessment of the ECM remodeling.

We have chosen two compounds, IBMX (non-selective phosphodiesterase inhibitor) and GM6001 (pan MMP inhibitor), as they have been shown to have a positive effect on remodeling biomarkers and are non-toxic in ex vivo models of the liver and cartilage $[12,19,20]$. The treatments were applied in what is generally accepted as the fibrotic phase, at day 14 following BLM administration.

We combined the rodent PCLS model with in vivo BLM treated lung tissue and the novel Protein Fingerprint biomarker technology for the assessment of the ongoing ECM protein remodeling. The aims of this study were to design and validate a model for screening of potential therapies for IPF.

\section{Methods \\ Reagents}

All reagents were standard high-quality chemicals from either Merck or Sigma-Aldrich. The culture medium William's Medium E was from Gibco (Life Technologies), while AlamarBlue assay was from AbD Serotec.

\section{Animal experiment}

Thirty female Sprague-Dawley rats, aged twelve weeks, were used for two experiments and were housed at the animal facilities at Nordic Bioscience, Denmark. The experiments were approved by the Animal Ethics Committee of the Danish Ministry of Justice (2011/561-2003, 2012-15-2934-00467). The rats were housed in standard cages, with bedding and nest material at $18-22{ }^{\circ} \mathrm{C}$ and fed with standard pellet diet and tap water ad libitum. The rats were kept under conditions of a $12 \mathrm{~h}$ light/dark cycle. Pulmonary fibrosis was induced by I.T. installation of two doses of BLM $(0.25 \mathrm{ml} / \mathrm{kg})$, two days apart. The BLM was sprayed into the lungs of the rats using the MicroSprayer ${ }^{\circ}$ Aerosolizer with the FMJ-250 High Pressure Syringe (Penn-Century, PA, USA). In the first experiment fourteen animals were given BLM and six animals were given saline. Ten animals were euthanized seven days after the last BLM/saline dose, and ten 
animals were euthanized fourteen days after the last $\mathrm{BLM} /$ saline dose.

In the second experiment seven animals were given BLM and three animals were given saline, two days apart. The animals were euthanized fourteen days after last BLM/saline dose.

\section{Ex vivo experiments}

Prior to euthanization of the rats, the animals were anesthetized and a small catheter was surgically inserted into the trachea. After euthanization, the catheter was used to fill the lungs with a low temperature gelling agarose in order to keep the lungs dilated. The lungs were excised and stored in ice-cold Krebs-Henseleit buffer containing $25 \mathrm{mM}$ glucose, $10 \mathrm{mM}$ HEPES and $25 \mathrm{mM}$ $\mathrm{NaHCO}_{3}$. PCLS were prepared from the lungs using a TSE Krumdieck tissue slicer MD 4000 as previously described [18]. The PCLS were cultured for $48 \mathrm{~h}$, as longer incubation would affect cell viability, at $37^{\circ} \mathrm{C}$ under carbogen atmosphere in sterile 48 well plates containing $300 \mu \mathrm{l}$ William's Medium E containing $25 \mathrm{mM}$ glucose, $50 \mu \mathrm{g} / \mathrm{mL}$ gentamicin and the respective treatment. Treatments used for the ex vivo model were $100 \mu \mathrm{M}$ IBMX (Sigma-Aldrich, Denmark), a non-selective PDE inhibitor, and $10 \mu \mathrm{M}$ GM6001 (Sigma-Aldrich, Denmark), a pan MMP inhibitor. After the culturing period the supernatants were collected and stored at $-20^{\circ} \mathrm{C}$ until use.

\section{Cell viability measured by AlamarBlue}

AlamarBlue is a sterile non-toxic aqueous oxidationreduction indicator that yields colorimetric changes and a fluorescent signal in response to metabolic activity. The active compound, resazurin, is an oxidation-reduction indicator that changes from the oxidized non-fluorescent form to the reduced fluorescent form according to the viability and proliferating activity of cells [21]. In short, $300 \mu \mathrm{L} 10 \%$ AlamarBlue was added to each well of the microtiter plate containing PCLS and incubated for $90 \mathrm{~min}$ at $37^{\circ} \mathrm{C}$ while shaking. The results were measured by an ELISA reader at $540-590 \mathrm{~nm}$.

\section{ELISA measurements}

The P1NP ELISA is specific for the N-terminal propeptide of type I collagen. It is a marker of collagen type I formation as the pro-peptide is cleaved off the mature protein prior to fibril assembly [22]. The C1M marker is specific for a fragment generated by MMP-2, -9 and -13 , and hence reflects MMP generated degradation of collagen type I [23]. The iP3NP marker is specific for an internal epitope of the N-terminal pro-peptide of type III collagen, which reflects the formation of collagen type III (unpublished). The C3M marker is specific for the a neo-epitope generated by MMP-9, and reflects the degradation of type III collagen [24]. The ELM7 marker is specific to a neo-epitope generated by MMP-7 degradation of elastin [25]. All the above ELISA assays are developed and validated by Nordic Bioscience (see references for the technical papers). The markers were assessed in the supernatants of the cultured PCLS, i.e. the release of the epitopes into the supernatants.

\section{Histological analysis}

Lung tissue were fixed in cold $4 \%$ neutral formalin diluted in phosphate-buffered saline at $4{ }^{\circ} \mathrm{C}$ and processed for paraffin embedding using a Sakura Tissue Tek VIP 5 Jr. Tissue Processor and cut on a microtome in $5 \mu \mathrm{m}$ thick slides.

\section{Sirius Red}

The slides were deparaffinized, rehydrated and incubated with Weigert's Haematoxylin for 8 min, washed in water, and then incubated with a $0.1 \%$ Sirius Red solution dissolved in aqueous saturated picric acid for $1 \mathrm{~h}$, washed in water, dehydrated and mounted with PERTEX ${ }^{\circ}$. Pictures were taken with an Olympus BX60 microscope mounted with an Olympus DP71 camera at 4x magnification.

\section{Orcein stain}

The slides were deparaffinized, rehydrated and incubated with Acid Orcein Solution for 30 min, washed in water, and then incubated with Mayer's Haematoxylin Solution for $30 \mathrm{~s}$, washed in water, dehydrated and mounted with PERTEX ${ }^{\circ}$. Pictures were taken with an Olympus BX60 microscope mounted with an Olympus DP71 camera at 40x magnification.

\section{Statistical analyses}

Results are shown as mean \pm standard error of mean (SEM). Differences between mean values were compared by nonparametric Mann-Whitney's $t$-test for two-tailed observations. All statistical analyses were performed in GraphPad Prism software v.6 (GraphPad Software, San Diego, CA). P values less than 0.05 were considered significant.

\section{Results}

\section{ECM remodeling is increased in fibrotic lung}

There was a 10-fold increase of type I collagen formation (P1NP) in the conditioned medium from BLM animals versus saline control animals at day 7 termination in the PCLS model $(p<0.0001)$ (Fig. 1a). Moreover P1NP was significantly increased in the conditioned medium from BLM animals at day 14 compared to day 14 saline controls $(p=0.0008)$, but significantly decreased when compared to day 7 BLM animals $(p<$ 0.0001) (Fig. 1a).

There were increased levels of type I collagen degradation $(\mathrm{C} 1 \mathrm{M})$ in the conditioned medium from BLM 


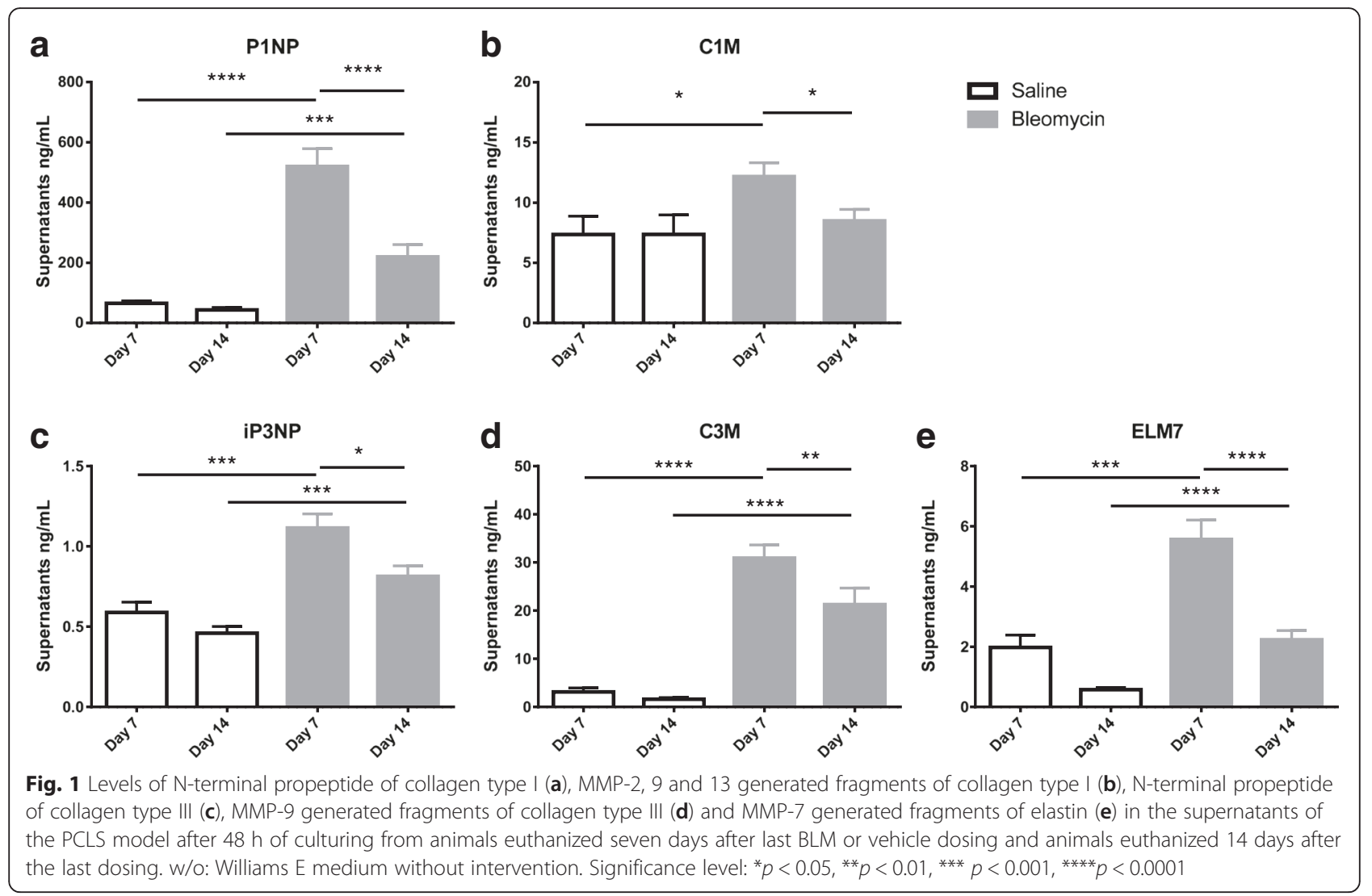

animals versus saline control animals in the PCLS model at day 7 termination $(p=0.016)$ (Fig. 1b). In addition, $\mathrm{C} 1 \mathrm{M}$ was significantly decreased in the conditioned medium of BLM animals terminated at day 14 when compared to conditioned medium from day 7 termination in the PCLS model ( $p=0.015)$ (Fig. 1b).

Type III collagen formation (iP3NP) was significantly increased in the conditioned medium from BLM animals when compared to saline control animals terminated at day 7 in the PCLS model $(p=0.0001)$ (Fig. 1c). The levels of iP3NP were significantly decreased in the conditioned medium from BLM animals terminated at day 14 when compared to the conditioned medium from BLM animals terminated day 7 in the PCLS model $(p=$ 0.017), but still significantly elevated compared to day 14 saline controls $(p=0.0005)$ (Fig. 1c).

There were a 15 -fold increase in the levels of collagen type III degradation $(\mathrm{C} 3 \mathrm{M})$ in the conditioned medium of BLM animals when compared to saline control animals terminated at day $7(p<0.0001)$ in the PCLS model (Fig. 1d). The levels of C3M were significantly decreased in the conditioned medium from BLM animals terminated at day 14 when compared to the conditioned medium from BLM animals terminated at day 7 in the PCLS model $(p=0.0008)$, but still significantly elevated compared to day 14 saline controls $(p<0.0001)$ (Fig. 1d).
Levels of MMP-7 generated elastin fragments (ELM7) were significantly increased the conditioned medium from BLM animals when compared to saline control animals terminated at day $7(p=0.0002)$ in the PCLS model (Fig. 1e). The levels of ELM7 were significantly decreased in the conditioned medium from BLM animals terminated at day 14 when compared to the conditioned medium from BLM animals terminated at day 7 $(p<0.0001)$ but still significantly elevated compared to day 14 saline controls $(p<0.0001)$ (Fig. 1e).

\section{Lung ECM remodeling can be differentially modulated by intervention}

There were significantly increased levels of type I collagen formation (P1NP) in the conditioned medium from BLM animals when compared to saline control animals $(p=0.013)$ in the PCLS model (Fig. 2a). Adding IBMX to the medium significantly decreased the levels $(p<$ 0.05), while adding GM6001 had no significant effect (Fig. 2a). There were significantly increased levels of type I collagen degradation $(\mathrm{C} 1 \mathrm{M})$ in the conditioned medium from BLM animals when compared to saline control animals $(p=0.014)$ in the PCLS model (Fig. 2b). Adding IBMX to the medium significantly decreased the levels $(p<0.0001)$, and the same was seen when adding GM6001 $(p<0.0001)$ to the medium (Fig. 2b). 


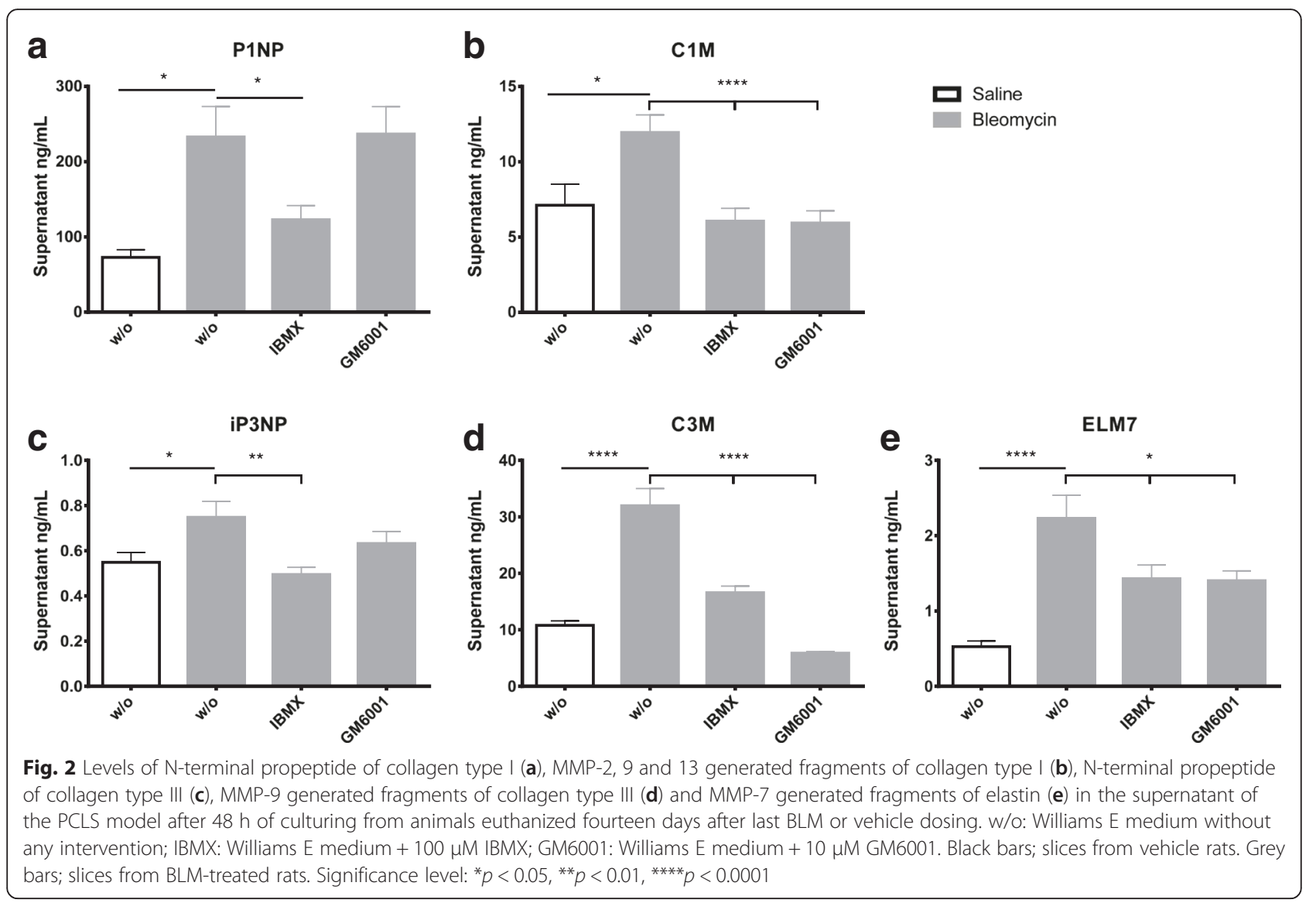

Significantly increased levels of collagen type III formation (iP3NP) were seen in the conditioned medium from BLM animals when compared to saline control animals $(p<0.05)$ in the PCLS model (Fig. 2c). Adding IBMX to the medium significantly decreased the levels $(p=0.003)$, while adding GM6001 had no significant effect (Fig. 2c).

Significantly increased levels of MMP-9 degraded collagen type III (C3M) were observed in the conditioned medium from BLM treated animals when compared to saline control animals $(p<0.0001)$ in the PCLS model (Fig. 2d). There was a 5-fold increase in the supernatant from the BLM treated animals, which could be significantly decreased by adding IBMX $(p<0.0001)$ to the medium, while GM6001 $(p<0.0001)$ was able to decrease the levels below vehicle (Fig. 2d).

Levels of MMP-7 generated fragments of elastin (ELM7) were significantly elevated in the conditioned medium from BLM treated animals when compared to vehicle treated animals $(p<0.0001)$ in the PCLS model (Fig. 2e). It was seen that adding IBMX $(p=0.02)$ and GM6001 $(p=0.015)$ were able to significantly decrease the ELM7 levels.

\section{Viability was sustained in the PCLS}

The viability of the PCLS was measured by AlamarBlue at baseline and after $48 \mathrm{~h}$ of culturing. Culturing for $48 \mathrm{~h}$ or adding IBMX or GM6001 to the lung slices had no effect on metabolic activity (small decrease, not significant) (Fig. 3).

\section{Histology}

Sirius Red staining was performed on the lung tissue. It was evident that collagen deposition was present in the lungs of the animals euthanized 7 and 14 days after last BLM dose compared to the saline control animals (Fig. 4). In addition, an Orcein Elastin Stain was performed on the lung tissue, and showed a higher presence of elastin fibers in the lungs of the animals euthanized 7 and 14 days after last BLM dose compared to the saline control animals (Fig. 5). The Orcein stain also clearly shows that the tissue sections coming from BLM rats are more nucleated.

\section{Discussion}

This is, to our knowledge, the first study to evaluate the fibrotic processes in the in vivo-like environment of the ex vivo PCLS system using novel formation and 

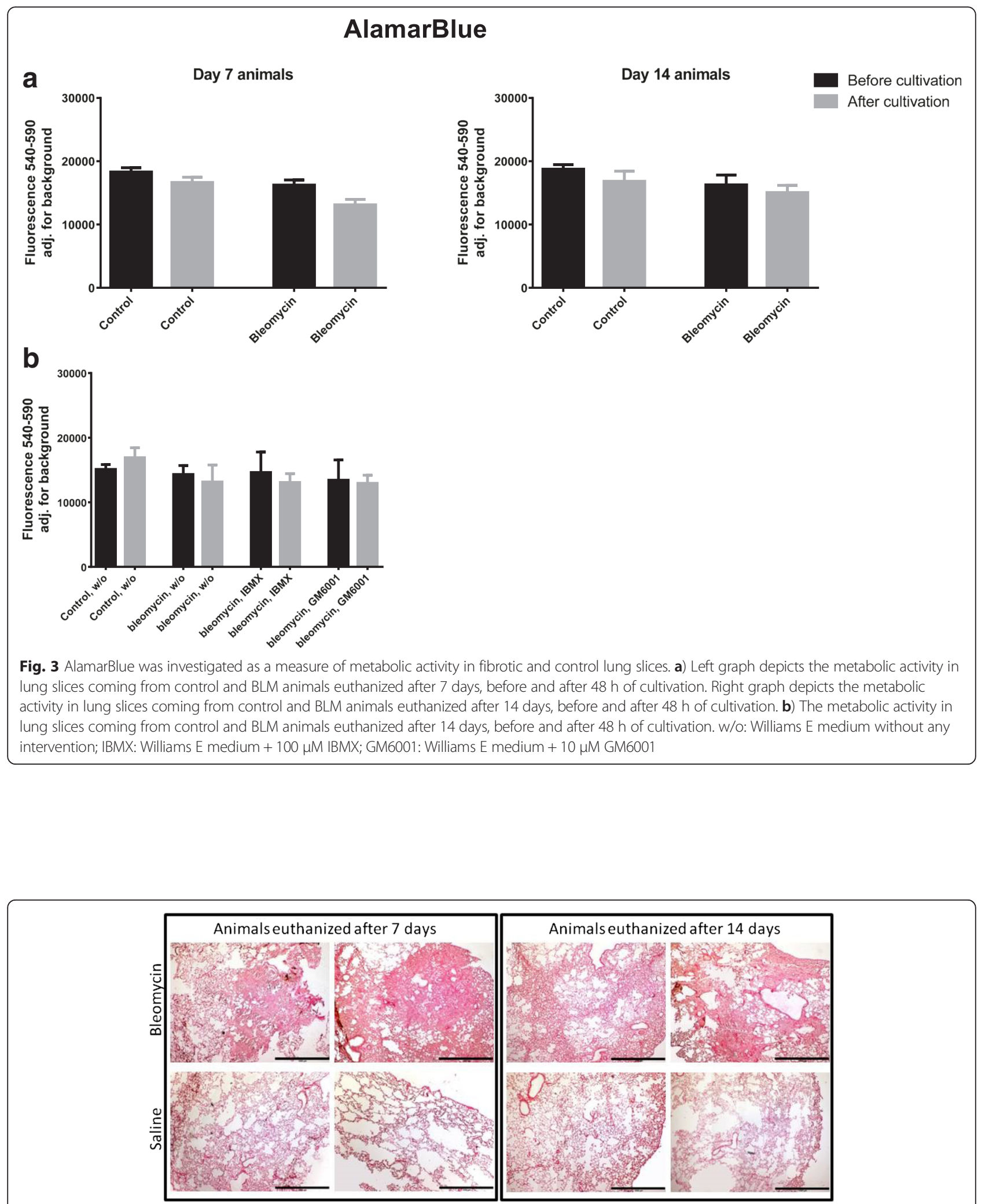

Fig. 4 Representative pictures of Sirius Red staining performed on lung tissue from saline control and BLM treated animals. The left section is slides from animals euthanized 7 days after last BLM or saline dose, while the right section is slides from animals euthanized 14 days after last BLM or saline dose. Slides from BML treated animals are depicted in the upper row, while slides from saline treated animals are depicted in the lower row. Pictures were taken at 4x magnification, and the black scale bar in the lower right corner of each picture indicates $1000 \mu \mathrm{m}$ 


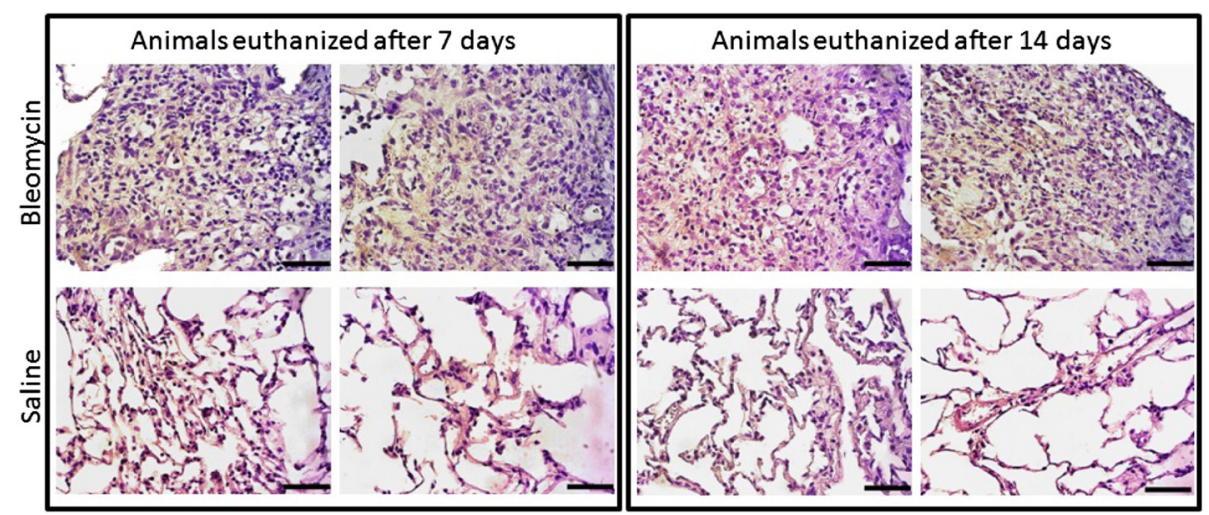

Fig. 5 Representative pictures of Orcein Elastin staining performed on lung tissue from saline control and BLM treated animals. Elastin appears brownish. The left section is slides from animals euthanized 7 days after last BLM or saline dose, while the right section is slides from animals euthanized 14 days after last BLM or saline dose. Slides from BML treated animals are depicted in the upper row, while slides from saline treated animals are depicted in the lower row. Pictures were taken at 40x magnification, and the black scale bar in the lower right corner of each picture indicates $100 \mu \mathrm{m}$

degradation markers of the ECM to investigate fibrosis remodeling. The animals used for the PCLS model were treated with BLM in vivo to induce a disease pattern resembling the IPF seen in human. The PCLS were then cultured in an ex vivo setup and the release of formation and degradation epitopes into the supernatant were assessed by ELISA based on monoclonal antibodies.

The highlighted results were: 1) significant differences were seen in the biomarkers levels between saline and BLM treated animals, 2) biomarker levels were inhibited by adding a phosphodiesterase inhibitor or a multiple MMP inhibitor to the culture medium, and 3) levels of the biomarkers decreased with increasing time between last in vivo BLM dosing and termination point.

Jenkins et al. recently published a study, in which they evaluated eleven different ECM biomarkers developed by Nordic Bioscience in the PROFILE IPF cohort [2]. Seven of these novel neoepitope biomarkers differed significantly in serum from patients diagnosed with IPF when compared to controls. In addition six of the neoepitope biomarkers were predictive of overall survival of the IPF patients. This paper clearly shows that the ECM remodeling taking place during the pathogenesis of IPF can be assessed by ECM biomarkers. In our rat PCLS model we have chosen ECM remodeling markers equivalent to the markers used by Jenkins et al.

The use of an PCLS model may be an important tool when evaluating ECM diseases such as IPF, since it is well known that cells cultured in traditional monolayer systems differentiate into a non in vivo-like phenotype, and may change protein expression profile [19, 26-28]. Another advantage of the PCLS model is the in vivo-like pathology kept within this system which may increase the disease relevance. This may improve the success rate when performing drug screening and raise the chance for a new treatment to reach and be implemented in the clinic. In the past decades over 200 drugs have been shown to be effective in the bleomycin model, with only 2 translating into therapeutic agents for IPF. The majority $(95 \%)$ of the drugs were given preventive ( $\leq 7$ days after last BLM dose), likely interfering with the inflammatory and early fibrogenic response [29, 30]. The US Food and Drug Administration (FDA) has stated that "Additional biomarkers and additional surrogate markers are needed to guide product development" [31]. The overall goal of using biomarkers is to improve decisionmaking with regards to dosing, toxicity and treatment time in order to save costs, which we hope can be applied in the model development.

It is well known that MMPs and other enzymes are upregulated in IPF and play an active role in matrix remodeling [32, 33]. This matrix remodeling can be assessed by biomarkers targeting the neoepitopes generated by MMP cleavage, in both formation and degradation of matrix proteins, thus the markers reflect the tissue balance of the ECM proteins. The results presented here indicate that multiple MMPs are upregulated and involved in the matrix remodeling occurring during disease. It was shown that the system used could be manipulated by adding treatments to the culture medium, suggesting that the model may be used as a system for testing ECM modifying drug candidates. It has previously been reported, within liver and cartilage ex vivo setups, that increasing cAMP levels (e.g. by addition of IBMX) affects ECM remodeling [12, 34]. The $\mathrm{C} 3 \mathrm{M}$ biomarker has shown to be applicable in a $\mathrm{CCl}_{4}$ liver fibrosis ex vivo setup. Veidal et al. showed that C3M was significantly elevated in supernatants from $\mathrm{CCl}_{4}$ treated animals when compared to vehicle animals. Furthermore by adding IBMX or GM6001 to the culture 
medium, they saw that the levels of C3M within the supernatants from $\mathrm{CCl}_{4}$ treated animals were restored back to the same levels as vehicle treated animals [12]. This indicates that biomarkers are useful in a precision-cut tissue slide (PCTS) setup and that these biomarkers can be inhibited by adding treatments to the culture medium.

It has generally been accepted that collagen type I and III are upregulated in lung tissue of IPF patients [32]. In this paper we show that the formation (P1NP) and degradation $(\mathrm{C} 1 \mathrm{M})$ of type I collagen are significantly increased in supernatants from BLM treated animals, when compared to saline treated animals. In the present study, the levels of both P1NP and C1M could be significantly inhibited by adding IBMX to culture medium, suggesting cAMP has a role in controlling type I collagen expression. The P1NP biomarker is not generated by MMPs, hence the addition of GM6001 did not have an effect on P1NP levels. However, the C1M biomarker is generated from cleavage by MMP-2, -9 and -13 , hence adding GM6001 to the culture medium significantly decreased the levels of C1M. Taken together these data suggest an increased turnover of type I collagen in the lungs of animals treated with BLM, as described in the literature but we are the first, as far as we know, to show this turnover in an ex vivo culture setup [2,32,35].

The levels of type III collagen formation (iP3NP) were significantly elevated in the supernatant of BLM treated animals, while IBMX was able to significantly decrease the levels. Again the iP3NP marker is not generated by MMP cleavage, hence GM6001 had no effect as expected. Our data showed that the degradation of type III collagen $(\mathrm{C} 3 \mathrm{M})$ in supernatants from BLM treated animals was 5-fold higher compared to supernatants from vehicle animals. The levels of C3M were significantly inhibited by adding IBMX or GM6001 to the culture medium. This is in accordance to what was seen in the ex vivo liver setup by Veidal et al. These data strongly indicate a higher collagen type III turnover in animals treated with BLM. In the PCLS model we are able to measure upregulated turnover levels of the two most abundant collagens, type I and III. As we are measuring in the supernatants from cultured lung slices, we know that the neo-epitopes are excreted from the lungs, and not subject to background from other parts of the body.

Elastin is a protein involved in the structure, function and elasticity of the ECM in the lungs [36]. MMP-7 is able to degrade elastin and high levels of this enzyme are associated with IPF $[37,38]$. ELM7 has previously been reported to be elevated in serum from IPF and lung cancer patients [25]. Our data showed that there are significantly increased levels of ELM7 in the supernatants from BLM treated animals. Adding IBMX could significantly decrease the levels, which confirms that increased cAMP levels can affect the MMP expression as seen by Karsdal et al. and Veidal et al [12, 34]. The ELM7 fragment is MMP generated and adding GM6001 to the culture medium could significantly decrease the levels as expected.

Several studies have shown that the BLM model is known to be reversible with time [29, 39-41], where sacrifice of the animals 14 days after a single I.T. dose of BLM was the most suitable. Chaudhary et al. saw that inflammatory markers are significantly increased during the first 9 days following BLM administration with resolution of the inflammation phase around day 9 [42]. The other interesting observation was the expression of pro-collagen 1 mRNA which begins at day 9 and then decreases back to baseline levels at day 21 . These observations lead to the speculation that the switch between inflammation and fibrosis in the rat BLM model occurs around day 9 [42]. Data in this paper shows that the collagen type 1 formation (P1NP) is significantly increased in the supernatants at day 7 following BLM administration when compared to vehicle animals. Interestingly, the levels of P1NP significantly decreases when comparing supernatants at day 14 to day 7 following BLM administration. However, the levels of P1NP in supernatants from BLM treated animals is still significant increased at day 14 following BLM administration when compared to supernatants from vehicle treated animals at day 14 . The degradation of type 1 collagen $(\mathrm{C} 1 \mathrm{M})$ was increased in supernatants from BLM treated animals compared to vehicle treated animals at day 7 . The levels of $\mathrm{C} 1 \mathrm{M}$ in supernatants from BLM animals decreased back to the same levels as saline treated animals at day 14. These data suggest that the type 1 collagen balance is shifted towards deposition of the collagen as observed by Chaudhary et al., but it should be noted that the model described in this paper is a double dose I.T. BLM given two days apart.

Shahzeidi et al. saw that levels of type III procollagen gene expression was increased in mice exposed to single I.T. dose of BLM. Van Hoozen et al. was able to measure elevated levels of type III procollagen mRNA as early as 4 days after administration of a single BLM I.T. dose in a rat model. Razzaque et al. saw a histological increase of type III collagen deposition over a 4 week period following a single BLM I.T. dose in a rat model [43-45]. Our data show that type III collagen formation is significantly elevated 7 days following BLM administration and then it significantly decreases 14 days following BLM administration when compared to day 7 . It should be noted that the levels at 14 days following BLM administration are still significantly elevated when compared to vehicle. This goes well with what was seen by Shahzeidi et al. and Van Hoozen et al., but contradicts the histological findings by Razzaque et al. 
To our knowledge the turnover of type III collagen in a BLM model has not been described. We saw the same pattern for degradation as with the formation of type III collagen except that there is a 15 -fold increase of C3M in supernatants from rats terminated 7 days following BLM administration.

To our knowledge the MMP degradation of ELM7 has not been described in a BLM model. Our findings follow what is seen with the formation of type I and III collagen. The levels are significantly elevated 7 days following BLM administration, which decreases significantly at 14 days following BLM administration when compared to day 7 . The levels are still significantly elevated at day 14 when compared to vehicle.

Lastly we show that the lung slices were viable after $48 \mathrm{~h}$ of culturing and that the given treatments did not affect the viability. Culturing of $48 \mathrm{~h}$ was chosen from previous experiments, showing that viability decreases rapidly after $48 \mathrm{~h}$ (data not shown). The histology shows a higher amount of collagen and elastin in the lungs of the animals that were given BLM compared to saline control animals, which correlate well with the data seen in the biomarker analyses.

\section{Conclusion}

We were able to generate a turnover profile in three of the most affected ECM proteins in IPF, collagen type I and III and elastin, in a BLM ex vivo model. By adding IBMX and GM6001 we have shown that we were able to significantly decrease the levels of the ECM biomarkers. We then showed that these ECM markers are significantly elevated 7 days following BLM administration and then significantly decreased 14 days after last BLM administration, but still significantly higher than their respective saline controls.

Understanding more on the biology and the interactions of all the cells in the tissue and by giving the treatment after the inflammatory phase, we believe that the risk of having false positives in a murine bleomycin model becomes less. The markers used in the PCLS setup are clinically validated markers shown to be upregulated in serum of IPF patients. This will increase the chances of translation from preclinical species to human, when screening for potential therapies.

\section{Abbreviations}

BLM, Bleomycin; ECM, Extracellular matrix; IBMX, 3-isobutyl-1-methylxanthine; IPF, Idiopathic pulmonary fibrosis; MMP, Matrix metalloproteinase; PCLS,

Precision-cut lung slices; PDE, Phosphodiesterase

\section{Funding}

This work was funded by "Den Danske Forskningsfond".

\section{Authors' contributions}

Author contributions: conception and design - NUBH, MK, SB, SC and DL Experimental work, analysis and interpretation - NUBH, MK, SB, SC, SR and
DL; Drafting of the manuscript and intellectual content - NUBH, MK, SB, SC, SR and DL. All authors read and approved the final manuscript.

\section{Competing interests}

N.U.B.H., M.K., S.R. and D.L. are full time employees of Nordic Bioscience A/S. Nordic Bioscience is a privately-owned; small-medium size enterprise (SME) partly focused on the development of biomarkers. S.B. and S.C. are full time employees of Grünenthal.

None of the authors received fees, bonuses or other benefits for the work described in the manuscript.

Morten A. Karsdal holds stocks in Nordic Bioscience A/S.

\section{Author details}

${ }^{1}$ Nordic Bioscience A/S, Herlev Hovedgade 205-207, 2730 Herlev, Denmark.

${ }^{2}$ University of Southern Denmark, SDU, Odense, Denmark. ${ }^{3}$ Grünenthal Group, Aachen, Germany.

Received: 24 March 2016 Accepted: 30 June 2016

Published online: 05 July 2016

\section{References}

1. Spagnolo P, Grunewald J, Bois RMD. Genetic determinants of pulmonary fibrosis: Evolving concepts. Lancet Respir Med. 2014;2:416-28. doi:10.1016/ S2213-2600(14)70047-5.

2. R.G. Jenkins, J.K. Simpson, G. Saini, J.H. Bentley, A.-M. Russell, R. Braybrooke, et al., Longitudinal change in collagen degradation biomarkers in idiopathic pulmonary fibrosis: an analysis from the prospective, multicentre PROFILE study, Lancet Respir. Med. (2015). doi:10.1016/S2213-2600(15)00048-X

3. N.U.B. Hansen, F. Genovese, D.J. Leeming, M.A. Karsdal, The importance of extracellular matrix for cell function and in vivo likeness., Exp. Mol. Pathol. (2015). doi:10.1016/j.yexmp.2015.01.006.

4. O'Brien LE, Jou TS, Pollack AL, Zhang Q, Hansen SH, Yurchenco P, et al. Rac1 orientates epithelial apical polarity through effects on basolateral laminin assembly. Nat Cell Biol. 2001;3:831-8.

5. Pedersen JA, Lichter S, Swartz MA. Cells in 3D matrices under interstitial flow: effects of extracellular matrix alignment on cell shear stress and drag forces. J Biomech. 2010:43:900-5.

6. Pedersen JA, Swartz MA. Mechanobiology in the third dimension. Ann Biomed Eng. 2005;33:1469-90.

7. Kirkpatrick CA, Selleck SB. Heparan sulfate proteoglycans at a glance. J Cell Sci. 2007:120:1829-32. doi:10.1242/jcs.03432.

8. Rozario T, DeSimone DW. The extracellular matrix in development and morphogenesis: a dynamic view. Dev Biol. 2010;341:126-40. doi:10.1016/j. ydbio.2009.10.026

9. Godbout C, Follonier Castella L, Smith EA, Talele N, Chow ML, Garonna A, et al. The mechanical environment modulates intracellular calcium oscillation activities of myofibroblasts. PLoS One. 2013;8:e64560. doi:10.1371/ journal.pone.0064560

10. Huang X, Yang N, Fiore VF, Barker TH, Sun Y, Morris SW, et al. Matrix stiffness-induced myofibroblast differentiation is mediated by intrinsic mechanotransduction. Am J Respir Cell Mol Biol. 2012;47:340-8. doi:10.1165/ rcmb.2012-00500C.

11. Li Z, Dranoff JA, Chan EP, Uemura M, Sévigny J, Wells RG. Transforming growth factor-beta and substrate stiffness regulate portal fibroblast activation in culture. Hepatology. 2007:46:1246-56. doi:10.1002/hep.21792.

12. Veidal SS, Nielsen MJ, Leeming DJ, Karsdal MA. Phosphodiesterase inhibition mediates matrix metalloproteinase activity and the level of collagen degradation fragments in a liver fibrosis ex vivo rat model. BMC Res Notes. 2012:5:686. doi:10.1186/1756-0500-5-686.

13. J.-P. Morin, J.-M. Baste, A. Gay, C. Crochemore, C. Corbière, C. Monteil, Precision cut lung slices as an efficient tool for in vitro lung physiopharmacotoxicology studies, Xenobiotica. (2012). http://www.tandfonline. com.proxy.findit.dtu.dk/doi/abs/10.3109/00498254.2012.727043\#.Vf7LI5es1LC (accessed September 20, 2015)

14. Park CC, Bissell MJ, Barcellos-Hoff MH. The influence of the microenvironment on the malignant phenotype. Mol Med Today. 2000;6: 324-9. doi:10.1016/S1357-4310(00)01756-1.

15. Schmeichel KL, Weaver VM, Bissell MJ. Structural Cues from the Tissue Microenvironment Are Essential Determinants of the Human Mammary Epithelial Cell Phenotype. J Mammary Gland Biol Neoplasia. 1998:3:201-13. doi:10.1023/a:1018751124382. 
16. Nelson CM, Bissell MJ. Of extracellular matrix, scaffolds, and signaling: tissue architecture regulates development, homeostasis, and cancer. Annu Rev Cell Dev Biol. 2006;22:287-309. doi:10.1146/annurev.cellbio.22.010305.104315.

17. Weigelt B, Bissell MJ. Unraveling the microenvironmental influences on the normal mammary gland and breast cancer. Semin Cancer Biol. 2008;18:311-21. doi:10.1016/j.semcancer.2008.03.013.

18. Westra IM, Pham BT, Groothuis GMM, Olinga P. Evaluation of fibrosis in precision-cut tissue slices. Xenobiotica. 2013;43:98-112. doi:10.3109/00498254. 2012.723151.

19. Sondergaard BC, Wulf $H$, Henriksen $K$, Schaller S, Oestergaard S, Qvist $P$, et al. Calcitonin directly attenuates collagen type II degradation by inhibition of matrix metalloproteinase expression and activity in articular chondrocytes. Osteoarthr Cartil. 2006;14:759-68. doi:10.1016/j.joca.2006.01.014.

20. Wang B, Chen P, Jensen A-CB, Karsdal MA, Madsen SH, Sondergaard B-C, et al. Suppression of MMP activity in bovine cartilage explants cultures has little if any effect on the release of aggrecanase-derived aggrecan fragments. BMC Res Notes. 2009;2:259. doi:10.1186/1756-0500-2-259.

21. Al-Nasiry S, Geusens N, Hanssens M, Luyten C, Pijnenborg R. The use of Alamar Blue assay for quantitative analysis of viability, migration and invasion of choriocarcinoma cells. Hum Reprod. 2007;22:1304-9. doi:10.1093/humrep/dem011.

22. Leeming DJ, Larsen DV, Zhang C, Hi Y, Veidal SS, Nielsen RH, et al. Enzymelinked immunosorbent serum assays (ELISAs) for rat and human $\mathrm{N}$-terminal pro-peptide of collagen type I (PINP)-assessment of corresponding epitopes. Clin Biochem. 2010;43:1249-56. doi:10.1016/j.clinbiochem.2010.07.025.

23. Leeming D, He Y, Veidal S, Nguyen Q, Larsen D, Koizumi M, et al. A novel marker for assessment of liver matrix remodeling: an enzyme-linked immunosorbent assay (ELISA) detecting a MMP generated type I collagen neo-epitope (C1M). Biomarkers. 2011;16:616-28. doi:10.3109/1354750X.2011. 620628

24. Barascuk N, Veidal SS, Larsen L, Larsen DV, Larsen MR, Wang J, et al. A novel assay for extracellular matrix remodeling associated with liver fibrosis: An enzyme-linked immunosorbent assay (ELISA) for a MMP-9 proteolytically revealed neo-epitope of type III collagen. Clin Biochem. 2010;43:899-904. doi:10.1016/j.clinbiochem.2010.03.012.

25. J.H. Kristensen, L. Larsen, B. Dasgupta, C. Brodmerkel, M. Curran, M.A. Karsdal, et al., Levels of circulating MMP-7 degraded elastin are elevated in pulmonary disorders., Clin. Biochem. (2015). doi:10.1016/j.clinbiochem.2015.07.009.

26. Hynes RO. Integrins: bidirectional, allosteric signaling machines. Cell. 2002; 110:673-87. http://www.ncbi.nlm.nih.gov/pubmed/12297042 (accessed January 14, 2015).

27. Hynes RO. The extracellular matrix: not just pretty fibrils. Science. 2009;326: 1216-9. doi:10.1126/science.1176009.

28. Ruiz PA, Jarai G. Discoidin domain receptors regulate the migration of primary human lung fibroblasts through collagen matrices. Fibrogenesis Tissue Repair. 2012;5:3. doi:10.1186/1755-1536-5-3.

29. Moeller A, Ask K, Warburton D, Gauldie J, Kolb M. The bleomycin animal model: a useful tool to investigate treatment options for idiopathic pulmonary fibrosis? Int J Biochem Cell Biol. 2008;40:362-82. doi:10.1016/j. biocel.2007.08.011.

30. Degryse AL, Lawson WE. Progress toward improving animal models for idiopathic pulmonary fibrosis. Am J Med Sci. 2011;341:444-9. doi:10.1097/ MAJ.0b013e31821aa000.

31. Karsdal MA, Henriksen K, Leeming DJ, Mitchell P, Duffin K, Barascuk N, et al. Biochemical markers and the FDA Critical Path: How biomarkers may contribute to the understanding of pathophysiology and provide unique and necessary tools for drug development. Biomarkers. 2009;14:181-202. doi:10.1080/13547500902777608.

32. Dancer RCA, Wood AM, Thickett DR. Metalloproteinases in idiopathic pulmonary fibrosis. Eur Respir J. 2011;38:1461-7. doi:10.1183/09031936.00024711.

33. Pardo A, Selman M. Role of matrix metaloproteases in idiopathic pulmonary fibrosis. Fibrogenesis Tissue Repair. 2012;5:S9. doi:10.1186/1755-1536-5-S1-S9.

34. Karsdal MA, Sumer EU, Wulf H, Madsen SH, Christiansen C, Fosang AJ, et al. Induction of increased CAMP levels in articular chondrocytes blocks matrix metalloproteinase-mediated cartilage degradation, but not aggrecanase-mediated cartilage degradation. Arthritis Rheum. 2007;56: 1549-58. doi:10.1002/art.22599.

35. Laurent GJ, Harrison NK, McAnulty RJ. The regulation of collagen production in normal lung and during interstitial lung disease. Postgrad Med J. 1988;64 Suppl 4:26-34. http://www.ncbi.nlm.nih.gov/pubmed/3076931 (accessed January 6, 2016).
36. Vrhovski B, Weiss AS. Biochemistry of tropoelastin. Eur J Biochem. 1998;258: 1-18. doi:10.1046/j.1432-1327.1998.2580001.x.

37. Fujishima S, Shiomi T, Yamashita S, Yogo Y, Nakano Y, Inoue T, et al. Production and activation of matrix metalloproteinase 7 (matrilysin 1) in the lungs of patients with idiopathic pulmonary fibrosis. Arch Pathol Lab Med. 2010;134:1136-42. doi:10.1043/2009-0144-OA.1.

38. Heinz A, Taddese S, Sippl W, Neubert RHH, Schmelzer CEH. Insights into the degradation of human elastin by matrilysin-1. Biochimie. 2011;93:187-94. doi:10.1016/j.biochi.2010.09.011.

39. Izbicki G, Segel MJ, Christensen TG, Conner MW, Breuer R. Time course of bleomycin-induced lung fibrosis. Int J Exp Pathol. 2002;83:111-9. http://www.pubmedcentral.nih.gov/articlerender. fcgi?artid=2517673\&tool=pmcentrez\&rendertype=abstract (accessed July 1, 2015).

40. Arizmendi N, Puttagunta L, Chung KL, Davidson C, Rey-Parra J, Chao DV et al. Rac2 is involved in bleomycin-induced lung inflammation leading to pulmonary fibrosis. Respir Res. 2014;15:71. doi:10.1186/1465-9921-15-71.

41. Schaefer CJ, Ruhrmund DW, Pan L, Seiwert SD, Kossen K. Antifibrotic activities of pirfenidone in animal models. Eur Respir Rev. 2011;20:85-97. doi:10.1183/09059180.00001111.

42. Chaudhary NI, Schnapp A, Park JE. Pharmacologic differentiation of inflammation and fibrosis in the rat bleomycin model. Am J Respir Crit Care Med. 2006;173:769-76. doi:10.1164/rccm.200505-7170C.

43. Shahzeidi S, Mulier B, de Crombrugghe B, Jeffery PK, McAnulty RJ, Laurent GJ. Enhanced type III collagen gene expression during bleomycin induced lung fibrosis. Thorax. 1993:48:622-8. http://www.pubmedcentral.nih.gov/ articlerender.fcgi?artid=464587\&tool=pmcentrez\&rendertype=abstract (accessed July 2, 2015).

44. Van Hoozen BE, Grimmer KL, Marelich GP, Armstrong LC, Last JA. Early phase collagen synthesis in lungs of rats exposed to bleomycin. Toxicology. 2000;147:1-13. doi:10.1016/S0300-483X(00)00142-6.

45. Razzaque M. Bleomycin-induced pulmonary fibrosis in rat is associated with increased expression of collagen-binding heat shock protein (HSP) 47. Virchows Arch Int J Pathol. 1998;432:455-60

\section{Submit your next manuscript to BioMed Central and we will help you at every step:}

- We accept pre-submission inquiries

- Our selector tool helps you to find the most relevant journal

- We provide round the clock customer support

- Convenient online submission

- Thorough peer review

- Inclusion in PubMed and all major indexing services

- Maximum visibility for your research

Submit your manuscript at www.biomedcentral.com/submit
) Biomed Central 